\title{
Effects of whole-body electromyostimulation on chronic nonspecific low back pain in adults: a randomized controlled study
}

This article was published in the following Dove Press journal: Journal of Pain Research

\author{
Anja Weissenfels' \\ Marc Teschler' \\ Sebastian Willert ${ }^{\prime}$ \\ Michael Hettchen' \\ Michael Fröhlich² \\ Heinz Kleinöder ${ }^{3}$ \\ Matthias Kohl ${ }^{4}$ \\ Simon von Stengel' \\ Wolfgang Kemmler' \\ 'Institute of Medical Physics, \\ Friedrich-Alexander University \\ of Erlangen, Erlangen, Germany; \\ ${ }^{2}$ Department of Sports Science, \\ University of Kaiserslautern, \\ Kaiserslautern, Germany; ${ }^{3}$ Institute \\ of Training Science and Sport \\ Informatics, German Sport University \\ Cologne, Cologne, Germany; \\ ${ }^{4}$ Department of Medical and Life \\ Sciences University of Furtwangen, \\ Villingen-Schwenningen, Germany
}

Correspondence: Anja Weissenfels Institute of Medical Physics, FriedrichAlexander University of Erlangen, Henkestrasse 91, Erlangen 91052, Germany

$\mathrm{Tel}+499$ |3| 8522840

Fax +49 9 | $3 \mid 8522824$

Email anja.weissenfels@imp.uni-erlangen. de
Purpose: Low back pain (LBP) is one of the most frequent chronic conditions worldwide. Data from a recent meta-analysis indicated that whole-body electromyostimulation (WB-EMS), a time-effective, joint-friendly, and highly individualized training technology, demonstrated promising effects on LBP; however, methodologic limitations prevent definitive evidence for this result. Thus, the aim of this study was to conduct a randomized controlled WB-EMS trial to determine the corresponding effect on chronic, nonspecific LBP in people with chronic LBP. Patients and methods: Thirty LBP patients, 40-70 years old, were randomly assigned into two groups (WB-EMS: 15; control [CG]: 15). While the nonactive CG maintained their lifestyle, the WB-EMS group completed a 12-week WB-EMS protocol $(1 \times 20 \mathrm{~min} /$ week $)$ with slight movements, specifically dedicated to LBP. Pain intensity and frequency were determined by a 4-week pain diary before and during the last 4 weeks of intervention. Primary study endpoint was average pain intensity at the lumbar spine.

Results: At baseline, no group differences apart from nonregular exercise were observed. Mean intensity of LBP decreased significantly in the WB-EMS group $(P=0.002)$ and remained unchanged in the $\mathrm{CG}(P=0.730)$, with a significant difference between both groups $(P=0.027)$. Maximum isometric trunk extensors improved significantly in the WB-EMS group $(P=0.005)$, while no significant difference was seen in the $\mathrm{CG}(P=0.683)$. In contrast to the significant difference between WB-EMS group and $\mathrm{CG}$ for the latter parameter $(P=0.038)$, no intergroup difference was determined for maximum isometric trunk flexors $(P=0.091)$. The WB-EMS group showed a significant increase of this parameter $(P=0.003)$, while no significant change was determined in the $\mathrm{CG}(P=0.563)$.

Conclusion: WB-EMS is a time-effective training method for reducing chronic nonspecific LBP and increasing maximum trunk strength in people with such complaints. After this promising comparison with a nonactive $\mathrm{CG}$, research needs to be extended to include comparisons with active groups (WB-Vibration, conventional back strengthening).

Keywords: electrical stimulation, electrical muscle stimulation, EMS, LBP, pain intensity, muscle strength

\section{Introduction}

Low back pain (LBP) is one of the leading orthopedic diseases worldwide and affects almost everyone at least once in their lives. ${ }^{1,2}$ In about $80 \%$ of cases, the cause of LBP is not known and is declared nonspecific. ${ }^{3}$ Physical exercise seems to be an effective conservative method to improve $\mathrm{LBP}^{4}$ However, nowadays a lack of time might be the main obstacle to exercise ${ }^{5}$; so many people may abstain from time-consuming exercise programs. Furthermore, "kinesiophobia", that is, the fear of movement because of pain, is common in LBP patients and impedes their taking up physical activity. ${ }^{6}$ 
A considerable number of studies have already confirmed the effect of training technologies like whole-body vibration on LBP and show positive results. ${ }^{7-10}$ Other novel methods, such as whole-body electromyostimulation (WB-EMS), have not been addressed by clinical trials, but two master's theses have addressed LBP in this context. ${ }^{11,12}$ Nevertheless, the time-effective character of WB-EMS with low subjective intensity seems to be an optimal option for people unmotivated or unable to exercise conventionally. In addition to the benefits mentioned above, the easy and joint-gentle low-intensity, low-amplitude exercises may also optimally respond to the "kinesiophobia" of LBP patients. Therefore, the aim of this study was to compare the effect of WB-EMS with a nonactive control group (CG) on chronic nonspecific LBP patients between 40 and 70 years. Our primary hypothesis was that, compared with the $\mathrm{CG}$, WB-EMS significantly decreases mean pain intensity at the lumbar spine (LS) in people with chronic nonspecific LBP. The secondary hypothesis was that, compared with the CG, WB-EMS increases maximum isometric strength in back and abdominal muscle in people with chronic nonspecific LBP.

\section{Patients and methods}

\section{Trial design}

The study was a randomized, controlled, 12-week parallelgroup trial with nonspecific chronic LBP patients 40-70 years old. In the present study, which constituted the initial phase of a multicenter project that compared the effects of WBEMS with recognized methods (WB-Vibration, conventional exercises interventions) of nonpharmacologic LBP therapy, we focus on the comparison between WB-EMS application and a non-training control. The study was planned, implemented, and conducted from February to July 2017 at the Institute of Medical Physics (IMP), Friedrich-Alexander University Erlangen-Nürnberg (FAU), Germany. The IMP was supported by the Pain Research Center Erlangen, both part of the University of Erlangen-Nürnberg (FAU) Germany. The study complied with the Helsinki Declaration "Ethical Principles for Medical Research Involving Human Subjects" and was approved by the Ethical Committee of the FAU (ethics application no. 224_15b). After detailed information, all study participants gave their written informed consent. The project was registered under DRKS00009528.

\section{Participants}

For the initial project phase, around 5,000 people, 40-70 years old living in the area of Erlangen, Germany, were contacted by personal letters using citizen registers provided by the municipality. Of importance, the letters already included the most important eligibility criteria (eg, LBP complaints). Three hundred fifty-one persons responded and were further assessed for eligibility. Subjects were included if they met the following criteria. 1) 40-70 years old; 2) characteristics of chronic LBP (50\% in the last 3 months); 3) nonspecific LBP (eg, no orthopedic diagnosis; average numerical rating scale $[\mathrm{NRS}] \geq 1$ ); 4) no continuous or very frequent use of analgesics ( $>4$ days/week); 5) no pharmacologic therapy or diseases affecting muscle metabolism (eg, glucocorticoids); 6) no conditions that prevent WB-EMS application (eg, epilepsy, cardiac pacemaker, thrombosis); and 7) absence of less than 2 weeks during the study period. Applying these criteria, 60 subjects ( 20 men, 40 women) were eligible and willing to participate in the study. Participants were randomly assigned to four intervention groups: WB-EMS ( $n=15)$, WBVibration $(n=15)$, conventional training $(n=15)$, and nonactive CG $(n=15)$ stratified by age $(40-55,56-70)$. However, in this contribution we focus on the comparison of WB-EMS group vs nonactive $\mathrm{CG}(\mathrm{n}=30$; nine men, 21 women). Figure 1 gives the participant flow through the study. Table 1 shows the baseline characteristics of the subjects of all groups.

\section{Intervention}

We contrasted a 12-week WB-EMS application to a nonactive $\mathrm{CG}$, without any further intervention. The intervention was conducted at the IMP, Erlangen, Germany, which is centrally located and can be easily reached by public transport. Certified trainers, who also recorded the participant's attendance and compliance, supervised and guided all the sessions. Apart from the study intervention, all the participants were requested to maintain their usual lifestyle.

\section{WB-EMS}

WB-EMS enables the simultaneous activation of up to eight to ten muscle groups (upper legs, upper arm, bottom, abdomen, chest, lower back, upper back, latissimus dorsi, and two free options; total stimulation area $2,800 \mathrm{~cm}^{2}$ ) with regionally dedicated intensity. ${ }^{13}$ We applied bipolar electric current with a frequency of $85 \mathrm{~Hz}$, an impulse width of $350 \mu \mathrm{s}$, a rectangular mode and an interval of 6 seconds stimulation and 4 seconds of rest once a week for 20 minutes. During the stimulation phase, participants performed low-amplitude movements specifically dedicated to LBP (Box 1). The participants completed one to three sets with six repetitions of six easy movements in a minor range of motion (eg, dynamic squatting with knee ankle $\geq 120^{\circ}$ ) to keep the effect of the voluntary exercise itself as low as possible. Once a week four 
All persons aged between 40 and 70 years living in the center of Erlangen were contacted by personalized letters with detailed study information $(n=5,000)$

The study was also advertised in a newsletter published by the University of Erlangen-Nürnberg

\section{I}

351 responded to the detailed cover letter

Eligibility was checked by phone interview and mail

\section{】}

\begin{tabular}{|c|}
\hline $\begin{array}{c}264 \text { subjects excluded by protocol: } \\
\text { Subjects with less back pain ( } \leq 50 \% \text { of the last } 3 \text { months): } n=67 \\
\text { Subjects with specific back pain: } n=72 \\
\text { Subjects with } \geq 2 \text { weeks of absence during intervention period: } n=32 \\
\text { Subjects with contraindication for WB-EMS (Pacemaker,TEP etc.): } n=45 \\
\text { Subjects with other diseases: } n=38 \\
\text { Subjects outside age range: } n=10\end{array}$ \\
\hline$\downarrow$ \\
\hline $\begin{array}{l}87 \text { subjects were invited to our lab for measurement } \\
\qquad \begin{array}{c}27 \text { subjects excluded by protocol: } \\
\text { Missing at test: } n=20 \\
\text { Quit shortly before: } n=7\end{array}\end{array}$ \\
\hline
\end{tabular}

】

60 subjects randomly allocated (stratified by age) to:

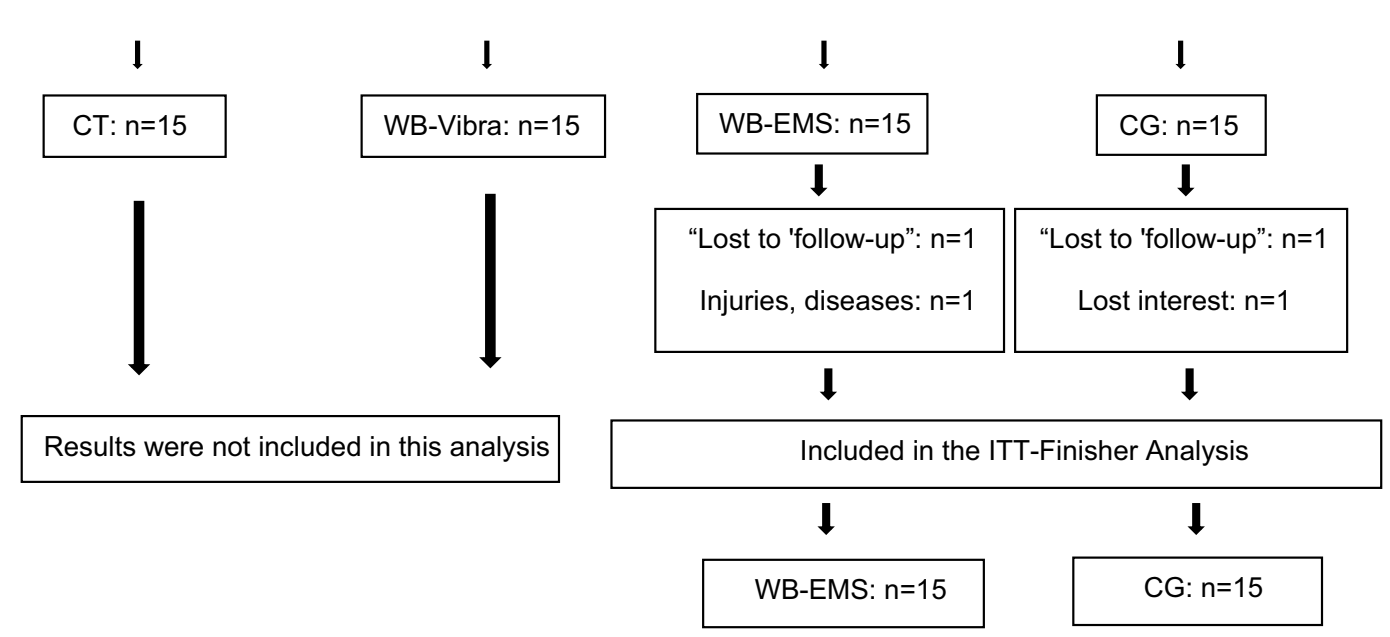

Figure I Flow diagram of the study intervention.

Notes: Due to low statistical power, study focus is on comparison of WB-EMS group with CG.

Abbreviations: CG, control group; CT, conventional back exercises; ITT, intent-to-treat; $n$, numbers; TEP, total endoprosthesis; WB-EMS, whole-body electromyostimulation; WB-Vibra, whole-body vibration. 
Table I Baseline characteristics of the WB-EMS group and control group

\begin{tabular}{|c|c|c|c|}
\hline Variable & WB-EMS $n=15$ & CG $n=15$ & $P$-value \\
\hline Age (years) ${ }^{\mathrm{a}}$ & $54.6 \pm 5.7$ & $59.4 \pm 7.7$ & 0.065 \\
\hline Gender $(M / F)^{a}$ & $6 / 9$ & $3 / 12$ & 0.247 \\
\hline Body height $(\mathrm{cm}), \mathrm{M} / \mathrm{F}^{\mathrm{b}}$ & $182 \pm 3 / 170 \pm 6$ & $175 \pm 5 / 166 \pm 8$ & $0.114 / 0.218$ \\
\hline Body mass $(\mathrm{kg}), \mathrm{M} / \mathrm{F}^{\mathrm{b}}$ & $92.3 \pm 17.7 / 77.0 \pm 20.3$ & $85.4 \pm 5.8 / 74.7 \pm 16.4$ & $0.438 / 0.781$ \\
\hline Total body fat (\%), M/Fb & $25.4 \pm 9.3 / 31.3 \pm 8.4$ & $29.9 \pm 5.4 / 35.5 \pm 9.1$ & $0.479 / 0.275$ \\
\hline RMDQ (number of items) ${ }^{\mathrm{a}, \mathrm{c}}$ & $4.8 \pm 2.6$ & $6.3 \pm 3.4$ & 0.201 \\
\hline Psych. factors (number of items) & $3.1 \pm 0.9$ & $3.2 \pm 1.3$ & 0.892 \\
\hline Acute use of analgesics $(n)^{a}$ & 3 & 5 & 0.426 \\
\hline No regular exercise $(n)^{a}$ & I & 6 & 0.031 \\
\hline Back pain-specific exercise $(n)^{a}$ & 10 & 8 & 0.322 \\
\hline Total exercise volume $(\mathrm{min} / \mathrm{w} .)^{\mathrm{a}}$ & $83.9 \pm 49.8$ & $85.6 \pm 43.8$ & 0.936 \\
\hline
\end{tabular}

Notes: ${ }^{a}$ Assessed by baseline questionnaire; bMeasured through Bio-Impedance Analysis (DSM-BIA, InBody 770, Seoul, Korea); 'RMDQ measured functional limitations due

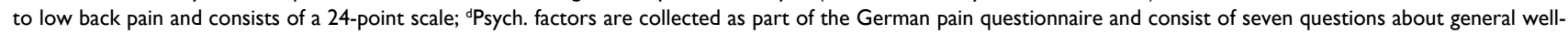
being ( 0 "does not apply at all" to 5 "fully apply").

Abbreviations: RMDQ, Roland and Morris Disability Questionnaire; psych., psychologic; WB-EMS, whole-body electromyostimulation; CG, control group; P, significance; M, male; F, female; w, week.

Box I Exercises of whole-body electromyostimulation intervention

\begin{tabular}{|l|}
\hline Exercises \\
\hline I. Squat with latissimus pulleys \\
2. Butterfly reverse (with angled arms) \\
3. Straight pullovers with trunk flexion (lumberjacks) \\
4. Standing trunk flexion (crunch) \\
5. One-legged stand with biceps curl \\
6. Side step with weight shift and biceps curl \\
\hline
\end{tabular}

Notes: Exercises consist of three sets of six repetitions with 6 seconds load and 4 seconds break (in total 20 minutes/unit). To get used to the electrical stimulation, a slow increase in time (12-20 minutes/unit) occurs in the first 4 weeks with only partial exercise sequence at the beginning of the intervention. The full exercise program was reached after 4 weeks.

subjects underwent video-guided exercise under the supervision of two certified instructors using WB-EMS devices from miha bodytec (Gersthofen, Germany). ${ }^{14}$

The intensity of the stimulation was regulated using the BORG CR 10 scale. ${ }^{15}$ Subjects were requested to exercise at a rate of perceived exertion (RPE) between "hard (5)" and "very hard (7)". In the first session current intensity was individually adapted in close interaction with the participants and saved to generate a fast and valid setting during the following sessions. The first four sessions' time was increased from 12 to 20 minutes with a time rise of 2 minutes per session. During each session, instructors slightly increased the intensity every 2-3 minutes in close cooperation with the individuals. Research assistants regularly monitored compliance with the prescribed exercise intensity.

\section{Control group}

The CG was asked to maintain its usual lifestyle. Participants of this group were regularly contacted by phone and asked about their current status and lifestyle changes. To maintain compliance with the $\mathrm{CG}$ protocol, participants of the $\mathrm{CG}$ were offered random allocation to one of the intervention groups (WB-EMS, WB-Vibration, conventional back exercises) of the second study period.

\section{Outcomes}

\section{Primary endpoint}

- Changes of average LBP intensity (4-week pain diary) from baseline to 12-week follow-up.

\section{Secondary endpoint}

- Changes of maximum isometric trunk extension from baseline to 12-week follow-up.

- Changes of maximum isometric trunk flexion from baseline to 12-week follow-up.

\section{Testing procedures}

The intervention took place between April 2017 and July 2017. Participants were assessed by the same research assistant at baseline and follow-up test at the same time of the day ( \pm 60 minutes).

\section{Anthropometry}

Height was determined barefoot to the nearest $0.1 \mathrm{~cm}$ with a stadiometer. Body mass and composition were determined through direct-segmental, multifrequency Bio-Impedance Analysis (DSM-BIA, InBody 770, Seoul, Korea). This device measures impedance of the trunk, arms, and legs separately using a tetrapolar eight-point tactile electrode system, which applies six frequencies between 1 and $1000 \mathrm{kHz}$. Participants were asked to avoid intense physical activity (eg, intensive 
endurance or resistance training) and to fast 3 hours prior to the assessments. Body mass index was calculated as body mass/body height $\left(\mathrm{kg} / \mathrm{m}^{2}\right)$.

\section{Back pain diary and questionnaire}

LBP intensity was monitored using a NRS from 0 (no pain) to 10 (worst possible pain) conducted 4 weeks before and during the last 4 weeks of the intervention. ${ }^{16}$ For this, participants were provided with standardized questionnaires and were requested to rate their highest daily LBP intensity. The average 4-week LBP intensity before and during the last 4 weeks of the intervention was included in the analysis as the primary endpoint. In parallel, the maximum daily pain intensity during the 4-week periods was determined by the identical procedure. Apart from the NRS, other tools for back pain research (German pain questionnaire, chronic pain grade [GCPS], Roland and Morrison Disability Questionnaire [RMDQ]) were applied; however, during the present first project phase we refrained from the analysis of these assessments. ${ }^{17-19}$ To detect factors that may confound our results, baseline and follow-up questionnaires monitored diseases, medication, lifestyle (changes), and adverse events related or unrelated to the intervention. The same baseline questionnaire asked for demographic parameters, education, family and social status, physical activity, psychosocial factors, and exercise. Research assistants carefully checked the completeness and accuracy of the baseline and follow-up questionnaires together with the participants.

\section{Functional testing}

In view of the focus on LBP, the functional testing concentrated on maximum isometric strength of trunk flexion and extension (Back-Check 607, Dr Wolff, Arnsberg, Germany). For the exact positioning and procedure, the assistants followed the manufacturer's recommendations. The adjustment of the devices was standardized for all patients. Patients were in a standing position $\left(0^{\circ}\right)$ with flexed knees $\left(20^{\circ}\right)$. Movement of the hip was fixed at the level of iliac crest back and front. For flexion, a pad was placed at the level of the sternum, while extension was measured at the scapula level. All patients completed each test three times; the highest result was included in the analysis. Applying this approach, reliability for maximum trunk extension (test-retest reliability; intra class correlation [ICC]) in this cohort was 0.88 (95\% CI: 0.82-0.93). A slightly lower ICC (0.86 [95\% CI: 0.81-0.90]) was determined for maximum trunk flexion.

\section{Sample size}

The sample size calculation of the study was based on the primary study endpoint "changes of LBP intensity". Based on previous research, we expected a standardized mean difference (SMD) between the WB-EMS group and CG of $0.45 \pm 0.45$ when applying the NRS $0-10$ scale. ${ }^{4,20}$ Thus, at least 16 participants per group were required to generate $\alpha=0.05$ and $\beta-1=80 \%$.

\section{Randomization}

Using age strata of 15 years (40-55, 46-70), eligible participants $(n=30)$ were randomly assigned $(1-1)$ to the study arms, WB-EMS and non-training CG (Figure 1). By drawing lots, participants allocated themselves in the study groups. Lots were placed in opaque plastic shells and drawn from a bowl. Neither participants nor the researchers knew the allocation beforehand. After balanced ( $n=15 /$ study arm) group allocation, the principal investigator (AW), responsible for the randomization process, instructed participants in detail about their status and corresponding dos and don'ts.

\section{Blinding}

Due to the inability to reliably blind participants, instructors, and primary researchers, we focused on blinding of the research assistants/outcome assessors concerning the group status of the participants. In other words, the outcome assessors were unaware whether subjects were allocated to the WB-EMS or CG and were not allowed to ask participants.

\section{Statistical analyses}

All the participants, who were randomly allocated, were included in the primary (intention to treat) analysis independent of compliance or loss to follow-up. R statistics software was used in combination with multiple imputation by Amelia II. ${ }^{21}$ The full data set was used for multiple imputation, with imputation being repeated 100 times. Overimputation diagnostic plots confirmed that the multiple imputations worked well in all cases. Based on a statistically (Shapiro-Wilk test) and graphically (QQ- and box-plots) checked normal distribution of the primary and secondary outcomes presented here, dependent $t$-tests were used to analyze within-group changes. Welch two-sample $t$-test was applied to determine differences between the groups. ${ }^{22}$ Chi-square tests were applied to detect difference in nominal scaled (baseline) data. Data were reported as mean value, SD, and 95\% CI. All tests were two-tailed, and statistical significance was accepted at $P<0.05$. Effect sizes (ESs) were calculated using 
Cohen's d. ${ }^{23}$ ES $\geq 0.5$ were considered as moderate; ES $\geq 0.8$ were considered as high. SPSS 25.0 (SPSS Inc, Chicago, IL, USA) was used for statistical procedures for baseline data.

\section{Results}

With the exception of no regular exercises, no significant differences were observed for baseline characteristics between WB-EMS group and CG (Table 1).

Based on 30 subjects randomized, two subjects, one in each group, were lost to follow-up. One woman of the WB-EMS group quit the trial during the first two weeks of intervention because of health problems unrelated to the study. The reason for one person dropping out from the $\mathrm{CG}$ is not known. Attendance rate of WB-EMS group was high and averaged $93 \% \pm 4 \%$; only one participant missed more than one WB-EMS session. Participants appropriately complied with the exercise protocol. With respect to exercise intensity, participants reported exercising within the given stipulation of RPE 5-7 at Borg CR10 RPE with a significant increase $(P=0.001)$ from $5.4 \pm 0.3$ in week 4 to RPE 5.9 \pm 0.5 in week $12 .{ }^{15}$ No adverse or unintended effects were observed during the WB-EMS session, and no participants reported any WB-EMS-related discomfort during or after WB-EMS application.

After 12 weeks of intervention, average daily LBP intensity monitored over 4 weeks $(P=0.028)$ changed significantly more favorably in the WB-EMS, compared with the $C G$ (ES: $d=0.754)$. In detail, while pain intensity decreased significantly in the WB-EMS group $(P=0.002)$, this parameter remained unchanged in the $\mathrm{CG}(P=0.730)$ (Table 2).

The maximum isometric trunk extensor (ie, lower back extensor) strength increased significantly by $14.6 \% \pm 18.6 \%$ in the WB-EMS group $(P=0.005)$ and was maintained in the CG $(-2.6 \% \pm 18.9 \%, P=0.683)$. The corresponding intergroup difference was significant $(P=0.038)$ with an ES that can be considered as "high". On the other hand, the effect for maximum isometric trunk flexor strength did not reach significance $(P=0.091)$. Within the WB-EMS group a significant increase of the maximum trunk flexion strength was determined $(15.3 \% \pm 17.8 \%, P=0.003)$, whereas no significant changes were observed in the CG $(4.0 \% \pm 17.3 \%, P=0.563)$.

No participant of the WB-EMS group or CG reported relevant changes of lifestyle including physical activity, exercise, diet, or general medication. However, the number of subjects with acute intake of analgesics was maintained in the $C G(n=5)$ and decreased from three to one person in the WB-EMS group.

\section{Discussion}

The primary aim of this study was to demonstrate the effect in the WB-EMS group vs a nonactive $\mathrm{CG}$ on chronic nonspecific LBP. In summary, our findings clearly indicate a significant positive effect on LBP in this mixed cohort 40-70 years old. Less consistently, significant effects in the WB-EMS group were determined for maximum isometric trunk extension but not for maximum isometric trunk flexion.

To our best knowledge to date, no other evidence-based trials have addressed the issue of WB-EMS effects on chronic LBP. There are only two nonpublished master's theses that evaluate the influence of WB-EMS on people with back-pain problems. ${ }^{11,12}$ Grützmacher (2003) reported a reduction of back pain in about $89 \%$ of the 49 participants after 6 weeks of $2 \times 45 \mathrm{~min} /$ week WB-EMS $(85 \mathrm{~Hz} ; 350 \mu \mathrm{s}$; 4 seconds load -2 seconds pause; bipolar). However, due to lack of a $\mathrm{CG}$, weak statistical procedures, and vague study endpoints, the scientific evidence of this study is quite restricted. After 6 weeks of WB-EMS application with the identical stimulation protocol as described above, Vatter (2010) reported a $92.3 \%$

Table 2 Changes of primary and secondary study endpoints for the WB-EMS group and CG

\begin{tabular}{|c|c|c|c|c|c|}
\hline & $\begin{array}{l}\text { WB-EMS } \\
M V \pm S D(P)\end{array}$ & $\begin{array}{l}\text { CG } \\
M V \pm S D(P)\end{array}$ & $\begin{array}{l}\text { Absolute difference } \\
\text { MV }(95 \% \mathrm{Cl})\end{array}$ & $P$-value & ES \\
\hline \multicolumn{6}{|c|}{ Average pain intensity ( 4 weeks) $[\text { Index }]^{a}$} \\
\hline Baseline & $2.75 \pm 1.46$ & $3.40 \pm 1.70$ & ----- & 0.274 & \\
\hline Difference & $-0.74 \pm 0.87^{* *}$ & $-0.08 \pm 0.88$ ns & $0.67(0.18$ to 1.24$)$ & 0.028 & 0.754 \\
\hline \multicolumn{6}{|c|}{ Maximum isometric trunk extension (kg) } \\
\hline Baseline & $49.60 \pm 19.08$ & $39.22 \pm 18.93$ & ----- & 0.150 & \\
\hline Difference & $7.26 \pm 9.69 * *$ & $-1.03 \pm 9.75 \mathrm{~ns}$ & $8.29(0.9$ to 16.4$)$ & 0.038 & 0.853 \\
\hline \multicolumn{6}{|c|}{ Maximum isometric trunk flexion (kg) } \\
\hline Baseline & $44.10 \pm 17.58$ & $31.96 \pm 17.02$ & ----- & 0.065 & \\
\hline Difference & $6.79 \pm 8.5 I^{* *}$ & $1.29 \pm 8.62$ ns & $5.5(-1.2$ to 12.0$)$ & 0.091 & 0.642 \\
\hline
\end{tabular}

Notes: andex from 0 (no pain) to 10 (worst possible pain); $* * P<0.01$; ${ }^{\text {ns }}$, nonsignificant.

Abbreviations: WB-EMS, whole-body electromyostimulation; $C G$, control group; $P$, significance; ES, effect size; MV, mean value. 
reduction of back pain in his WB-EMS group $(n=119)$. Comparing the WB-EMS group with the CG $(n=5)$, the author reported a significant positive effect of WB-EMS on back pain $(P<0.001)$. Nevertheless, in both studies intervention groups are not clearly limited to participants with nonspecific LBP. ${ }^{11,12}$

Confirming the data of a recent meta-analysis (WB-EMS vs $C G$ ) of individual patient data, the SMD of -0.84 is slightly higher than in the present study (SMD: -0.75$).{ }^{20}$ We attribute this difference predominantly to the different methodology of the studies. While the participants of the meta-analysis had to state their back pain retrospectively for the last week, the present study used a much more reliable 4-week pain diary. Further, the cohort of the present study was much more homogeneous (chronic, nonspecific, LS) with respect to LBP.

Comparing the study results with conventional exercise programs in the area of LBP, the average SMD reported by a recent meta-analysis (0.32) is below the effect of the present study. ${ }^{4}$ Closer to WB-EMS training, strength and resistance training trials demonstrated an average SMD of 0.51; however, the results for the individual exercise trials vary considerably. Reviewing the most effective exercise studies, two of which applied strength and resistance exercise, and one a core stabilization protocol, they reported very impressive effects on chronic LBP. ${ }^{24-26}$ Both resistance exercise training protocols, a periodized whole-body strength training with free weights (16 weeks, 3 units/week, 12-15 exercises, three sets with 8-12 repetitions at $53 \%-72 \% 1$ repetition maximum), and closely supervised, isolated back-extension exercise training on a specific back device (MEDX Lumbar Extension Machine, MedX, Ocala, Florida; one unit/week, one set with $80 \% 1$ repetition maximum, full range of motion vs limited range of motion [50\%]) resulted in nearly twice as high effects as the present study. However, the most striking effect (SMD: 2.27) came from a core stabilization program (4 weeks; $3 \times 30 \mathrm{~min} /$ week). ${ }^{26}$ Despite the high effects generated by these exercise studies, their exercise programs either required moderate-high training volume, or prescribed high exercise intensities. However, as discussed above, both aspects conflict with the lack of time and "kinesiophobia" of many participants with LBP.

Although we did not observe a difference between WBEMS group and $\mathrm{CG}$ for maximum isometric trunk flexion, the significant changes of maximum isometric strength in the back $(+14.6 \% \pm 18.6 \% ; P=0.005)$ and abdomen $(+15.3 \% \pm 17.8 \%$, $P=0.003)$ were impressive. Only a few studies with emphasis on LBP include a measurement of maximum trunk extensors and flexors and none of them applied a similar device (Dr Wolff Back-Check, Arnsberg, Germany), which renders a comparative discussion difficult. Including a comparable cohort, after 6 weeks of isolated lumbar extension resistance training, Steele et al generated an increase of isometric trunk extension up to $54 \%{ }^{24}$ However, the training and test exercise were identical, and thus the results might be partially biased by a habituation effect. A similar isolated back-extension program conducted with active US soldiers without LBP $(n=298)$ demonstrated an increase of $13.3 \%$ for isometric back-extension strength. ${ }^{27} \mathrm{~A}$ study with conventional back exercises (stabilization vs dynamic; two sessions per week; 60 minutes; 8 weeks) reported back extension strength changes of between $16.3 \%$ and $48.5 \%\left(0^{\circ}-72^{\circ}\right.$ lumbar flexion $)$, whereby the stabilization group performed significantly better than the dynamic one. ${ }^{28}$ However, with an average age of 28.5 \pm 4.9 years, the cohort is also much younger than in the present study, which may explain the difference in strength gain. A similar cohort $(52.3 \pm 12.3$ years $)$ with LBP was measured after classical outpatient rehabilitation (ie, exercises, manual therapy, massage, and electrotherapy). ${ }^{29}$ After 6 months of intervention, LS extension strength increased by $18.2 \%$, while lumbar flexion rose by $16.7 \%$. While these results were comparable to the present study, the time efficiency of this trial (42.6 \pm 2.3 sessions in 6 months) is much less favorable. Thus, although there might be exercise protocols similar or slightly more effective than our protocol, the comparative advantage of WB-EMS vs conventional exercise strategies is its timeeffective, joint-friendly, and highly customized application. Further, by applying a minimum degree of common sense and the proper application of recently published WB-EMS guidelines, WB-EMS is a very safe exercise technology even eligible for multimorbid older people. ${ }^{14,30,31}$

However, some limitations might decrease the scientific evidence and generalizability (ie, external validity) of our study results. 1) The sample size of 15 per group is slightly lower than that suggested by power analysis $(n=16)$ based on a recent meta-analysis of individual patient data. ${ }^{20} 2$ ) Although this LBP project focuses on a comprehensive evaluation of different types of exercise (WB-EMS vs WBVibration vs back-strengthening exercise), the sample size generated in the first study phase did not allow a meaningful statistical comparison of the treatment groups. 3) As mentioned above, average intensity (5.9 RPE) was reported at an average of between 5 "hard" and 7 "very hard". Compared with other WB-EMS studies, a slightly lower intensity was specified in order to prevent possible "kinesiophobia" and a certain caution in electrical applications, especially in this critical group. To what extent this relatively low-intensity protocol optimally addresses chronical nonspecific back pain 
is still open to question and requires further studies. 4) In this study we determined a positive effect of WB-EMS on chronic nonspecific LBP in 40-70-year-old people with corresponding complaints. Thus, with respect to generalization, we conclude that our results are transferable to the majority of people with chronic nonspecific LBP. However, our results did not cover acute nonspecific LBP, which is still listed as a relative contraindication by the manufacturer (miha bodytec). In addition, the effects of WB-EMS on specific back pain are very difficult to grasp, as there are too many different causes with different responses to the electrical impulses. 5) Despite the specification to refrain from changes of lifestyle or physical activity habits during the intervention period, three participants of the CG started new treatments (acupuncture, osteopathy, and physical activity/ exercise). However, no CG participant finished a complementary therapy. In contrast, no changes were reported by the WB-EMS group.

\section{Conclusion}

In summary, WB-EMS is a time-effective, safe, and jointfriendly option for people unable or unmotivated to exercise conventionally. Due to a supervised WB-EMS training, especially participants with "kinesiophobia" get an optimal support without being afraid of movement load. Due to the rather small sample size generated during the first study phase, emphasis of this contribution was placed on the comparison with a nonactive CG. By increasing the number of participants, our further research will focus on the comparison of WB-EMS with established training options for LBP (WB-Vibration, conventional back exercises).

\section{Acknowledgment}

This publication is part of the doctoral work for the title "Dr rer. biol. hum."

\section{Disclosure}

The authors report no conflicts of interest in this work.

\section{References}

1. GBD 2013 DALYs and HALE Collaborators, Murray CJ, Barber RM, Dalys GBD, et al. Global, regional, and national disability-adjusted life years (DALYs) for 306 diseases and injuries and healthy life expectancy (HALE) for 188 countries, 1990-2013: quantifying the epidemiological transition. Lancet. 2015;386(10009):2145-2191.

2. Schmidt CO, Raspe H, Pfingsten M, et al. Back pain in the German adult population: prevalence, severity, and sociodemographic correlates in a multiregional survey. Spine. 2007;32(18):2005-2011.

3. Abraham I, Killackey-Jones B. Lack of evidence-based research for idiopathic low back pain: the importance of a specific diagnosis. Arch Intern Med. 2002;162(13):1442-1444.
4. Searle A, Spink M, Ho A, Chuter V. Exercise interventions for the treatment of chronic low back pain: a systematic review and meta-analysis of randomised controlled trials. Clin Rehabil. 2015;29(12):1155-1167.

5. Korsch S, Herbold D, Wiezoreck M, et al. Promoting factors, barriers and barrier management to the implementation of health-promoting behavior among rehabilitative patients with chronic low back pain - a qualitative analysis. Rehabilitation. 2016;55(4):210-216.

6. Lüning BC, Lundberg M, Lindberg P, Elfving B. Change in kinesiophobia and its relation to activity limitation after multidisciplinary rehabilitation in patients with chronic back pain. Disabil Rehabil. 2012;34(10):852-858.

7. Boucher JA, Abboud J, Dubois JD, Legault E, Descarreaux M, Henchoz Y. Trunk neuromuscular responses to a single whole-body vibration session in patients with chronic low back pain: a cross-sectional study. J Manipulative Physiol Ther. 2013;36(9):564-571.

8. Iwamoto J, Takeda T, Sato Y, Uzawa M. Effect of whole-body vibration exercise on lumbar bone mineral density, bone turnover, and chronic back pain in post-menopausal osteoporotic women treated with alendronate. Aging Clin Exp Res. 2005;17(2):157-163.

9. Kaeding TS, Karch A, Schwarz R, et al. Whole-body vibration training as a workplace-based sports activity for employees with chronic lowback pain. Scand J Med Sci Sports. 2017;27(12):2027-2039.

10. del Pozo-Cruz B, Hernández Mocholí MA, Adsuar JC, Parraca JA, Muro I, Gusi N. Effects of whole body vibration therapy on main outcome measures for chronic non-specific low back pain: a single-blind randomized controlled trial. J Rehabil Med. 2011;43(8):689-694.

11. Vatter J. Elektrische Muskelstimulation als Ganzkörpertraining - Multicenterstudie zum Einsatz von Ganzkörper-EMS im Fitness-Studio. München: AVM-Verlag; 2010.

12. Boeckh-Behrens W, Grützmacher N, Sebelefsky J. Elektromyostimulationstraining mit dem BodyTransformer - eine erfolgreiche Maßnahme zur Reduzierung von Rückenbeschwerden. Bayreuth: Institute of Sport Science; 2002.

13. Kemmler W, von Stengel S. Whole-body electromyostimulation as a means to impact muscle mass and abdominal body fat in lean, sedentary, older female adults: subanalysis of the TEST-III trial. Clin Interv Aging. 2013;8:1353-1364.

14. Kemmler W, Froehlich M, von Stengel S, Kleinöder H. Whole-body electromyostimulation - the need for common sense! Rationale and guideline for a safe and effective training. Deutsche Zeitschrift für Sportmedizin. 2016;2016(09):218-221.

15. Borg E, Kaijser L. A comparison between three rating scales for perceived exertion and two different work tests. Scand J Med Sci Sports. 2006;16(1):57-69.

16. von Korff M, Jensen MP, Karoly P. Assessing global pain severity by self-report in clinical and health services research. Spine. 2000;25(24):3140-3151.

17. Klasen BW, Hallner D, Schaub C, Willburger R, Hasenbring M. Validation and reliability of the German version of the Chronic Pain Grade Questionnaire in primary care back pain patients. Psychosoc Med. 2004; 1:(Doc07).

18. Casser HR, Hüppe M, Kohlmann T, et al. German pain questionnaire and standardised documentation with the KEDOQ-Schmerz. A way for quality management in pain therapy. Schmerz. 2012;26(2): $168-175$.

19. Roland M, Morris R. A study of the natural history of back pain. Part I: development of a reliable and sensitive measure of disability in lowback pain. Spine. 1983;8(2):141-144.

20. Kemmler W, Weissenfels A, Bebenek M, et al. Effects of whole-body electromyostimulation on low back pain in people with chronic unspecific dorsal pain: a meta-analysis of individual patient data from randomized controlled WB-EMS trials. Evid Based Complement Alternat Med. 2017;2017:8480429.

21. Honaker J, King G, Blackwell M. Amelia II: a program for missing data. JSS. 2011;45(7):1-47.

22. Barnard J, Rubin DB. Miscellanea. Small-sample degrees of freedom with multiple imputation. Biometrika. 1999;86(4):948-955. 
23. Cohen J. Statistical Power Analysis for the Behavioral Sciences. 2nd ed. Hillsdale, NJ: Lawrence Erlbaum Associates; 1988.

24. Steele J, Bruce-Low S, Smith D, Jessop D, Osborne N. A randomized controlled trial of limited range of motion lumbar extension exercise in chronic low back pain. Spine. 2013;38(15):1245-1252.

25. Kell RT, Asmundson GJ. A comparison of two forms of periodized exercise rehabilitation programs in the management of chronic nonspecific low-back pain. $J$ Strength Cond Res. 2009;23(2):513-523.

26. Cho HY, Kim EH, Kim J. Effects of the CORE exercise program on pain and active range of motion in patients with chronic low back pain J Phys Ther Sci. 2014;26(8):1237-1240.

27. Mayer JM, Childs JD, Neilson BD, Chen H, Koppenhaver SL, Quillen WS. Effect of lumbar progressive resistance exercise on lumbar muscular strength and core muscular endurance in soldiers. Mil Med. 2016;181(11):e1615-e1622.
28. Moon HJ, Choi KH, Kim DH, et al. Effect of lumbar stabilization and dynamic lumbar strengthening exercises in patients with chronic low back pain. Ann Rehabil Med. 2013;37(1):110-117.

29. Alfuth M, Welsink DW. Pain and functional outcomes after outpatient physiotherapy in patients with low back pain. Orthopade. 2017;46(6):522-529.

30. Kemmler W, Teschler M, Weissenfels A, et al. Whole-body electromyostimulation to fight sarcopenic obesity in community-dwelling older women at risk. Results of the randomized controlled FORMOsAsarcopenic obesity study. Osteoporos Int. 2016;27(11):3261-3270.

31. Kemmler W, Weissenfels A, Teschler M, et al. Whole-body electromyostimulation and protein supplementation favorably affect sarcopenic obesity in community-dwelling older men at risk: the randomized controlled FranSO study. Clin Interv Aging. 2017;12:1503-1513.
Journal of Pain Research

\section{Publish your work in this journal}

The Journal of Pain Research is an international, peer reviewed, open access, online journal that welcomes laboratory and clinical findings in the fields of pain research and the prevention and management of pain. Original research, reviews, symposium reports, hypothesis formation and commentaries are all considered for publication.

\section{Dovepress}

The manuscript management system is completely online and includes a very quick and fair peer-review system, which is all easy to use. Visit http://www.dovepress.com/testimonials.php to read real quotes from published authors. 